\title{
Computational analysis of the electromechanical performance of mitral valve cerclage annuloplasty using a patient-specific ventricular model
}

\author{
Kyung Eun Lee', Ki Tae Kim', Jong Ho Lee', Sujin Jung ${ }^{2}$, June-Hong Kim ${ }^{2 * *}$, and Eun Bo Shim ${ }^{1, *}$ \\ ${ }^{1}$ Department of Mechanical and Biomedical Engineering, Kangwon National University, Chuncheon 24340, ${ }^{2}$ Department of Cardiology, College of Medicine, \\ Pusan National University, Busan 46241, Korea
}

\author{
ARTICLE INFO \\ Received September 5, 2018 \\ Revised October 15, 2018 \\ Accepted October 18, 2018

\section{*Correspondence \\ June-Hong Kim \\ E-mail: junehongk@gmail.com \\ Eun Bo Shim \\ E-mail: ebshim@kangwon.ac.kr}

\section{Key Words}

Lumped parameter model

Mitral valve cerclage annuloplasty

Patient-specific model

Ventricular electromechanical model
ABSTRACT We aimed to propose a novel computational approach to predict the electromechanical performance of pre- and post-mitral valve cerclage annuloplasty (MVCA). Furthermore, we tested a virtual estimation method to optimize the left ventricular basement tightening scheme using a pre-MVCA computer model. The present model combines the three-dimensional (3D) electromechanics of the ventricles with the vascular hemodynamics implemented in a lumped parameter model. $3 \mathrm{D}$ models of pre- and post-MVCA were reconstructed from the computed tomography $(C T)$ images of two patients and simulated by solving the electromechanicalgoverning equations with the finite element method. Computed results indicate that reduction of the dilated heart chambers volume (reverse remodeling) appears to be dependent on ventricular stress distribution. Reduced ventricular stresses in the basement after MVCA treatment were observed in the patients who showed reverse remodeling of heart during follow up over 6 months. In the case who failed to show reverse remodeling after MVCA, more virtual tightening of the ventricular basement diameter than the actual model can induce stress unloading, aiding in heart recovery. The simulation result that virtual tightening of the ventricular basement resulted in a marked increase of myocardial stress unloading provides in silico evidence for a functional impact of MVCA treatment on cardiac mechanics and post-operative heart recovery. This technique contributes to establishing a pre-operative virtual rehearsal procedure before MVCA treatment by using patient-specific cardiac electromechanical modeling of pre-MVCA.

\section{INTRODUCTION}

Conventional mitral valve annuloplasty is very effective for repairing mitral valve regurgitation. However, it is an invasive treatment employing open-heart surgery with sternotomy and thoracotomy. Mitral valve cerclage annuloplasty (MVCA) is a newly introduced catheter-based procedure used to treat heart failure patients without dangerous invasive surgery [1,2]. Since the MVCA technique avoids the clinical risks of the open-heart procedure, this method is less invasive, less risky and more accessible than conventional mitral valve annuloplasty. This procedure tightens the mitral annular circumference, inspired from epicardial purse-string annuloplasty [2,3]. Patients treated by this tightening procedure benefit from the stress unloading effect in their ventricular muscles, which ultimately reduces the size of the ventricles. Successful MVCA cases in swine, canine and human were reported previously [2-4]. However, the clinical outcomes of the MVCA procedure were not satisfactory for some patients [2of the Creative Commons Attribution Non-Commercial License, which permits unrestricted non-commercial use, distribution, and reproduction in any medium, provided the original work is properly cited. Copyright @ Korean J Physiol Pharmacol, pISSN 1226-4512, elSSN 2093-3827
Author contributions: E.B.S. provided the design of this research. K.E.L. analyzed and interpreted the data. K.E.L. wrote the main manuscript and E.B.S. revised the manuscript. K.T.K. and J.H.L. assisted for simulations. J.H.K. and S.J.J. provided the clinical data. J.H.K. discussed about clinical-related issues. All authors reviewed this manuscript. E.B.S. and J.H.K. contributed equally as corresponding authors. 
4]. Moreover, questions concerning the physiological mechanism involved in heart-muscle recovery due to this procedure and how much mitral circumference needs to be tightened depending on the patient remain unanswered. Therefore, for this MVCA procedure to be performed on heart-failure patients, the physiological mechanism induced by the MVCA procedure must be delineated in detail, and a patient-specific application method of the procedure needs to be established.

Considering the many difficulties in performing an experimental or a clinical study using a living heart, computer simulation can be an alternative method to understand the physiological mechanism of MVCA treatment. Until now, computer simulation of cardiac electrophysiology and mechanics has been considered a significant tool for the investigation of the heart disease mechanism and for the development of novel therapeutic clinical techniques for heart diseases [5-7]. For example, multiscale heart modeling was developed by integrating cell, tissue, and organ scales to investigate the electrophysiological behavior of the heart [8-12]. However, most of the computational studies on the mechanism of heart disease have been performed not on a patient-specific model, but rather on a typical cardiac model [5-10]. On the other hand, in the case of MVCA simulation, a patient-specific model is inevitable because tightening of mitral circumference can vary depending on the patient.

In this study, we propose a patient-specific cardiac electromechanical model of pre- and post-MVCA treatment to analyze the physiological mechanism of MVCA treatment. Using this model, the MVCA simulations of two typical patients were conducted, and the effects of MVCA treatment on cardiac mechanics and heart recovery were investigated. Furthermore, we demonstrate that the computational model presented herein can be used as a virtual test tool of MVCA therapy before a real clinical trial.

\section{METHODS}

\section{Clinical characteristics of MVCA}

In MVCA treatment, tensioning material surrounds the basement of the left ventricle (LV) (Fig. 1) [2]. A circumferential cerclage suture transverses the coronary sinus and the basal septal myocardium and is secured within the right atrium. In Fig. 1, the planes of the mitral annulus and MVCA are shown in blue and red, respectively.

Patients with severe heart failure underwent MVCA treatment in 2015 and 2016 at Pusan National University Yangsan Hospital (Korea) [4]. The study protocol was approved by the Korea Food and Drug Administration and by the institutional ethics board of Pusan National University Yangsan Hospital (NCT02471664) [4]. Among them, two representative patients were selected for computer simulation. Both patients showed significant reduction of mitral regurgitation after MVCA, the patient of case 1 showed significant recovery of all clinical symptom and sign. The patient of case 2 didn't show significantly reduction of dilated left ventricle chamber volume defined as 'reverse remodeling'. Computed tomography (CT) images for pre- and post-MVCA were scanned for each patient. A 6-month follow-up CT scan was obtained after MVCA in the patient with evident reverse remodeling finding. This study was approved by the Institutional Review Board of the hospital. Clinical characteristics of the patients are summarized in Table 1.

\section{Computational model of MVCA}

For the computer simulation of MVCA, we first constructed patient-specific ventricular geometry based on the CT images (Fig. 2). Next, 3D cardiac models of pre- and post-MVCA for each patient were established by applying electromechanical-governing
A

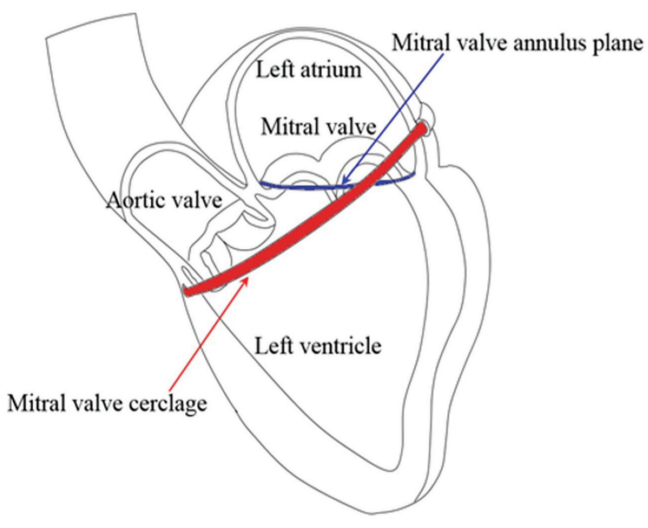

B

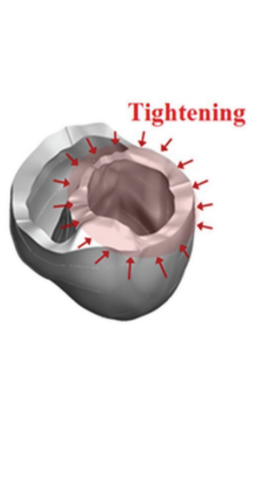

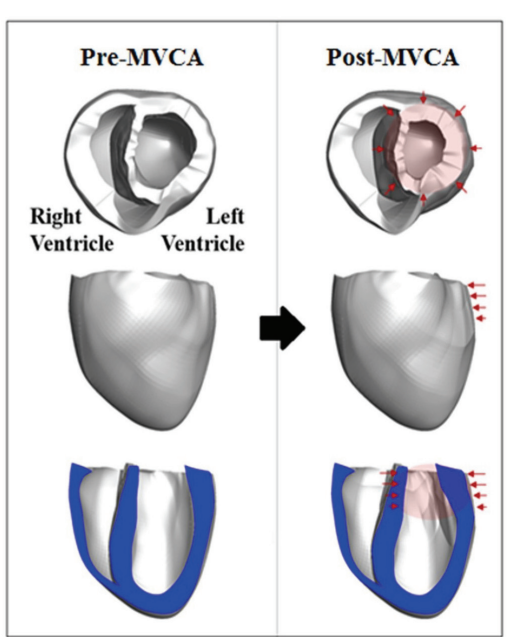

Fig. 1. Ventricular electromechanical model of MVCA. (A) Schematic of mitral valve cerclage annuloplasty. (B) Pre- and post-MVCA models of the electromechanical modeling of patient-specific ventricles. 
Table 1. Clinical characteristics of the patients

\begin{tabular}{lcc}
\hline \multicolumn{1}{c}{ Characteristic } & Case I & Case II \\
\hline Age (years) & 76 & 68 \\
Weight $(\mathrm{kg})$ & 51.9 & 68 \\
Height $(\mathrm{cm})$ & 154.9 & 167.5 \\
Pre-MVCA BP (mmHg) & $110 / 70$ & $100 / 66$ \\
$\quad$ SBP/DBP & & \\
Post-MVCA 1M FU & $91 / 58$ & $96 / 63$ \\
$\quad$ BP (mmHg) SBP/DBP & & \\
Pre-MVCA CT-based EF (\%) & 57 & 32 \\
Post-MVCA 1M FU EF & 61 & 34 \\
$\quad$ Echo/CT-based EF (\%) & & \\
Post-MVCA 6M FU EF & 62 & \\
$\quad$ Echo/CT-based EF (\%) & & \\
Clinical outcome & Good (Recovered) & Not Recovered \\
\hline
\end{tabular}

BP, SBP, and DBP represent blood pressure, systolic blood pressure, and diastolic blood pressure, respectively. In the table, 'month' is abbreviated ' $\mathrm{M}$ ' and 'follow-up' is abbreviated 'FU.' Thus, $1 \mathrm{M} \mathrm{FU}$ and $6 \mathrm{M}$ FU represent 1-month follow-up and 6-month follow-up, respectively. EF represents ejection fraction.

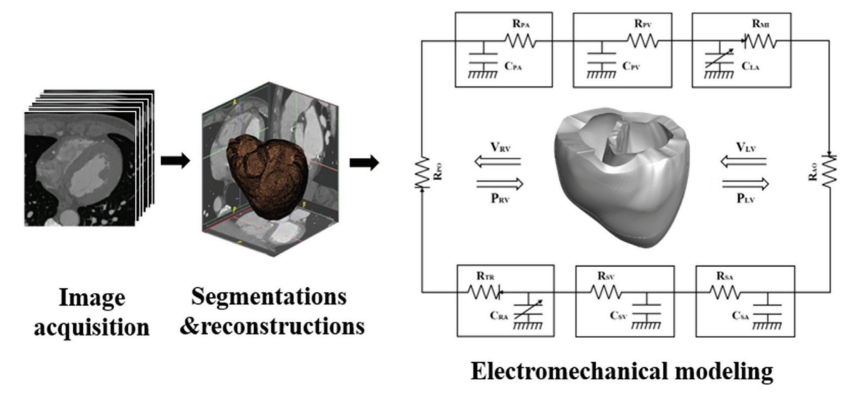

Fig. 2. Schematic diagram of the 3D finite-element ventricular electromechanical model coupled with the circulatory model. $P_{\mathrm{RV}}$, right ventricular pressure; $V_{R v}$, right ventricular volume; $P_{\mathrm{LV}}$, left ventricular pressure; $V_{\mathrm{L} v}$, left ventricular volume; $R_{\mathrm{PA},}$ pulmonary artery resistance; $C_{P A}$, pulmonary artery compliance; $R_{P V}$, pulmonary vein resistance; $C_{P V}$ pulmonary vein compliance; RMI, forward resistance of mitral valve; $C_{L A}$, left atrial compliance; $R_{A O}$ forward resistance of aortic valve; $R_{S A}$ systemic artery resistance; $C_{S A}$ systemic artery compliance; $R_{S V}$ systemic vein resistance; $C_{S v}$, systemic vein compliance; $R_{T R}$, tricuspid valve resistance; $C_{R A}$, right atrium compliance; and $R_{P U}$, pulmonary valve resistance.

equations to the model geometries.

In this study, we used the cardiac electromechanical model (Fig. 2) proposed in our previous paper [5,6]. This model integrates two main components: electrical and mechanical components of the ventricles. Here, the vascular hemodynamics implemented in the lumped parameter model (LPM) interacts with the ventricular mechanics. The myocardium in the ventricular mechanics model was assumed to be an orthotropic, hyperelastic, nearly incompressible material defined by an exponential strain energy function [5]. The mechanics model comprised 356 nodes and 172 elements in terms of Hermite polynomial interpolation, and was solved by using the finite element method. The electrical activa-
Table 2. Estimated physiological parameters for the patientspecific lumped parameter model in cases I and II

\begin{tabular}{lccccc}
\hline & \multicolumn{2}{c}{ Case I } & & \multicolumn{2}{c}{ Case II } \\
\cline { 2 - 3 } \cline { 5 - 6 } & $\mathrm{R}$ & $\mathrm{C}$ & $\mathrm{R}$ & $\mathrm{C}$ \\
\hline Pulmonary artery & 0.006 & 4.300 & & 0.006 & 4.300 \\
Pulmonary vein & 0.009 & 8.400 & & 0.009 & 8.400 \\
Systemic artery & 1.000 & 1.600 & & 1.000 & 2.080 \\
Systemic vein & 0.050 & 100.000 & & 0.050 & 100.000 \\
Aorta & 0.0120 & - & & 0.006 & - \\
\hline
\end{tabular}

Units of resistance $(\mathrm{R})$ and capacitance $(\mathrm{C})$ are $\mathrm{mmHg} \cdot \mathrm{s} / \mathrm{ml}$ and $\mathrm{ml} / \mathrm{mmHg}$, respectively.

tion times in cardiac tissue were obtained by solving the finite element equations of the mono-domain formulation, as described in our previous paper $[5,6]$ and were mapped to the mechanics mesh system of the ventricles.

The transient calcium concentration in cardiac cells was obtained from the cellular electrophysiological model. This calcium activation induced a force generation mechanism of cross-bridge dynamics in cardiac tissue myofilaments [13]. Actively generated cellular forces were integrated in the ventricular mechanics model. The mechanical behavior of the ventricular muscles was governed by the stress equilibrium equations based on the large deformation theory [5,6]. An LPM was coupled with the electromechanical model to present time-varying changes in circulatory hemodynamics coupled with the 3D electromechanical model (Fig. 2). Tissue remodeling of the ventricles associated with heart failure was implemented by changing the related parameters of the electromechanical model, as described in our previous paper [6].

\section{RESULTS}

In the present study, we simulated two patients with the physiological characteristics shown in Table 1. For Case I, simulations for pre-MVCA, and 1-month and 6-months follow-up after MVCA were performed. These three models were reconstructed from the respective CT images. In Case II, the clinical outcome after MVCA was not good, and only the 1-month follow-up data were gathered. Although the reason for the bad clinical outcome of Case II is not known, we suspect that the heart was too late to be brought up to recovery. To show an example of virtual surgical test for Case II, a simulation model with a tighter degree of LV circumference was also computed.

\section{Validation of the present method}

To validate the present model, we compared the computed variables with clinically measured data. In the present method, there are several model parameters that should be determined 
according to the patient's physiological state. The coefficient of cellular force generation of the cardiac mechanics model and the resistance and capacitance values of the LPM are examples. We set these model parameters so that the computed systole blood pressure (SBP), diastole blood pressure (DBP) and ejection fraction (EF) of the pre-MVCA model matched the measured values of the patient's pre-MVCA state. The selected simulation parameter of LPM, obtained from pre-MVCA are shown in Table 2. Next, we compared the simulated results with the measured data in terms of end-diastole volume (EDV), end-systole volume (ESV) and mid-diastole volume (MDV). In Case I (Table 3), the computed EDV and MDV values of pre-MVCA agreed well with the measured data (error less than 8\%), but the computed ESV had an error of approximately $12 \%$ compared with the measured value. Using the same lumped parameters obtained from pre-MVCA, we performed the post-MVCA simulation for the patient (1-month $[\mathrm{M}]$ follow-up [FU] and 6M FU) and compared the computed SBP, DBP and EF values with the measured data. In the case of

Table 3. Comparison of computed results with measured values in case $I$

\begin{tabular}{cccc}
\hline & Pre-MVCA & $\begin{array}{c}\text { Post-MVCA } \\
(1 \mathrm{M} \mathrm{FU})\end{array}$ & $\begin{array}{c}\text { Post-MVCA } \\
(6 \mathrm{M} \mathrm{FU})\end{array}$ \\
\hline SBP $(\mathrm{mmHg})$ & & & \\
Measured & 110 & 91 & $(\mathrm{~N} / \mathrm{A})$ \\
Computed & & 110 & 91 \\
Error $(\%)$ & $(\mathrm{N} / \mathrm{A})$ & 20.8 & $(\mathrm{~N} / \mathrm{A})$ \\
DBP $(\mathrm{mmHg})$ & & & \\
Measured & 70 & 58 & $(\mathrm{~N} / \mathrm{A})$ \\
Computed & & 69 & 58 \\
Error $(\%)$ & $(\mathrm{N} / \mathrm{A})$ & 18.9 & $(\mathrm{~N} / \mathrm{A})$ \\
EF $(\%)$ & & & \\
Measured & 57 & 61 & 62 \\
Computed & & 53.76 & 61.33 \\
Error $(\%)$ & $(\mathrm{N} / \mathrm{A})$ & 11.8 & 1.08 \\
EDV (ml) & & & \\
Measured & 169 & 154 & 106 \\
Computed & 167.64 & 141.60 & 100.51 \\
Error $(\%)$ & 0.80 & 8.06 & 5.18 \\
ESV (ml) & & & \\
Measured & 72 & 60 & 40 \\
Computed & 80.91 & 65.61 & 38.87 \\
Error $(\%)$ & 12.375 & 9.35 & 2.83 \\
MDV (ml) & & & 75 \\
Measured & 150 & 126 & 80.56 \\
Computed & 138.03 & 116.82 & \\
Error $(\%)$ & 7.98 & 7.29 & \\
\hline Computed & & & \\
\hline
\end{tabular}

Computed results from the patient-specific pre-MVCA model, post-MVCA $1 \mathrm{M}$ FU model and post-MVCA 6M FU model in Case I. $1 \mathrm{M} \mathrm{FU}$ and $6 \mathrm{M}$ FU represent the 1-month follow-up and 6-month follow-up, respectively. SBP, DBP, EDV, ESV, MDV, and EF represent systolic blood pressure, diastolic blood pressure, pulse pressure, end-diastolic left ventricular volume, end-systolic left ventricular volume, mid-diastolic left ventricular volume, and ejection fraction, respectively.
$1 \mathrm{M} \mathrm{FU}$, the computed $\mathrm{EF}$ value had $11 \%$ error, but the computed SBP and DBP values had approximately $20 \%$ error relative to the measured values (Table 3). In the case of $6 \mathrm{M} \mathrm{FU}$, the measured SBP and DBP values were not available, and only the measured EF value was compared with the computed one. The computed EF value of $6 \mathrm{M} \mathrm{FU}$ (Case I) agreed well with the measured data (1.08\% error). The computed EDV, ESV and MDV values of $1 \mathrm{M}$ FU and $6 \mathrm{M}$ FU had errors less than $10 \%$ compared with the measured values.

Table 4 shows the comparison between the measured data and the computed values for Case II. The computed EDV, ESV, and MDV values of pre-MVCA had errors less than $5 \%$ compared with the measured data. The computed EDV, ESV, and MDV values of pre-MVCA agreed well with the measured values (within $\sim 5 \%$ error range). For the post-MVCA model of the Case II, only the $1 \mathrm{M}$ FU data were compared. The computed SBP and DBP values had relatively large errors of $13.5 \%$ and $19.04 \%$, respectively. However, the computed EF value was within the error range ( 8\%). In the cases of EDV, ESV and MDV values, the computed data

Table 4. Comparison of computed results with measured values in case II

\begin{tabular}{cccc}
\hline & Pre-MVCA & $\begin{array}{c}\text { Post-MVCA } \\
(1 \mathrm{M} \mathrm{FU})\end{array}$ & $\begin{array}{c}\text { Virtual } \\
\text { Post-MVCA }\end{array}$ \\
\hline SBP $(\mathrm{mmHg})$ & & & \\
Measured & 100 & 96 & $(\mathrm{~N} / \mathrm{A})$ \\
Computed & & 109 & 114 \\
Error $(\%)$ & $(\mathrm{N} / \mathrm{A})$ & 13.54 & $(\mathrm{~N} / \mathrm{A})$ \\
DBP $(\mathrm{mmHg})$ & & & \\
Measured & 66 & 63 & $(\mathrm{~N} / \mathrm{A})$ \\
Computed & & 75 & 78 \\
Error $(\%)$ & $(\mathrm{N} / \mathrm{A})$ & 19.04 & $(\mathrm{~N} / \mathrm{A})$ \\
EF $(\%)$ & & & \\
Measured & 32.17 & 34 & $\mathrm{~N} / \mathrm{A}$ \\
Computed & & 31.21 & 36.75 \\
Error $(\%)$ & $(\mathrm{N} / \mathrm{A})$ & 8.20 & $(\mathrm{~N} / \mathrm{A})$ \\
EDV (ml) & & & \\
Measured & 286 & 273 & $(\mathrm{~N} / \mathrm{A})$ \\
Computed & 281.72 & 261.31 & 231.26 \\
Error $(\%)$ & 1.50 & 4.28 & $(\mathrm{~N} / \mathrm{A})$ \\
ESV (ml) & & & \\
Measured & 194 & 181 & $(\mathrm{~N} / \mathrm{A})$ \\
Computed & 198.70 & 179.75 & 146.27 \\
Error $(\%)$ & 2.42 & 0.69 & $(\mathrm{~N} / \mathrm{A})$ \\
MDV (ml) & & & \\
Measured & 269 & 240 & $(\mathrm{~N} / \mathrm{A})$ \\
Computed & 255.31 & 236.92 & 207.2 \\
Error $(\%)$ & 5.09 & 1.28 & $(\mathrm{~N} / \mathrm{A})$ \\
\hline Compur & & & \\
\hline
\end{tabular}

Computed results for the patient-specific pre-MVCA model, postMVCA 1M FU model and virtual post-MVCA model in Case II. $1 \mathrm{M}$ FU represents the 1-month follow-up. SBP, DBP, EDV, ESV, MDV, and EF represent systolic blood pressure, diastolic blood pressure, pulse pressure, end-diastolic left ventricular volume, end-systolic left ventricular volume, mid-diastolic left ventricular volume, and ejection fraction, respectively. 
of $1 \mathrm{M}$ FU agreed well with the measured values within the error range of $5 \%$ (Table 4 ).

\section{Simulation of the patient-specific model}

Transient variations in LV pressure and volume, as well as aortic blood pressure, were calculated from the patient-specific ventricular simulations of the two patients (Figs. 3 and 4). In Case I, the LV and aortic pressures did not change much after $1 \mathrm{M} \mathrm{FU}$ of post-MVCA, but remarkably decreased after $6 \mathrm{M}$ FU of postMVCA. The LV volume of pre-MVCA also decreased gradually after 6M FU. Case II underwent MVCA treatment, but the clinical outcome was not good. As shown in the upper panel of Fig. 4, the LV and aortic blood pressures did not change after MVCA. Additionally, an extremely overloaded volume of the patient's LV did not decrease, even after MVCA treatment. In the case of the virtual test with a tighter degree of LV circumference, the LV volume decreased remarkably, showing the relief of volumeoverloaded LV hypertrophy.
To evaluate the effect of stress unloading in post-MVCA ventricles, we analyzed the 'von-Mises stress' (hereafter, 'stress') distribution of the myocardium. Fig. 5 shows how end-diasystolic stress distribution changed in pre- and post-MVCA for Cases I and II. The left panel of Fig. 5 represents the spatial distribution of stress exerted on the ventricular muscle at the end of diasystole in Case I. In this case, the high-stress region of pre-MVCA in the ventricular basement was reduced after $1 \mathrm{M} \mathrm{FU}$ of post-MVCA. A mean stress loaded over the ventricular muscle tissue was also significantly reduced after $6 \mathrm{M}$ FU of MVCA, from $95 \mathrm{kPa}$ to 87 $\mathrm{kPa}$. Interestingly, the high-stress region of pre-MVCA in the ventricular apex nearly disappeared after $6 \mathrm{M} \mathrm{FU}$, indicating recovery of both ventricles.

In Case II, a poor clinical outcome was observed in spite of marked reduction of mitral regurgitation, and the heart did not recover from intractable heart failure despite MVCA treatment. Interestingly, the high-stress region area increased significantly at the basement and the middle of the ventricle after MVCA (right panel of Fig. 5). Furthermore, the mean stress load on the

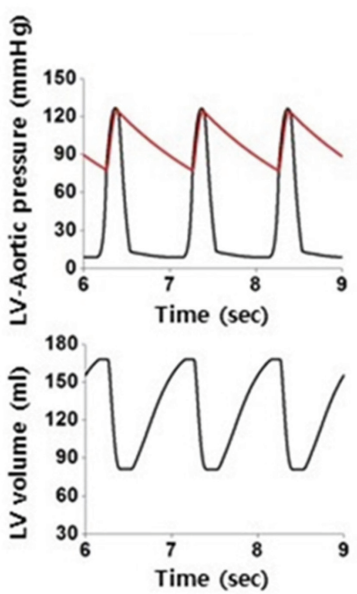

Pre-MVCA

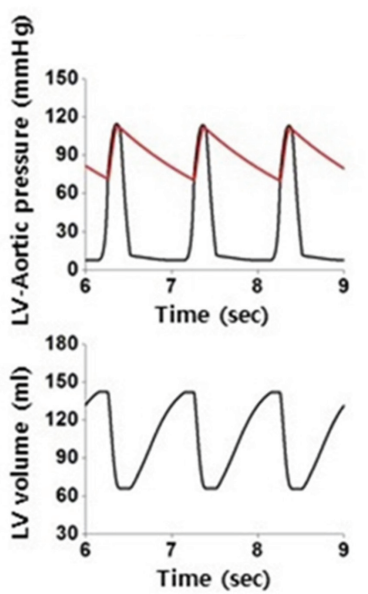

Post-MVCA (1M FU)

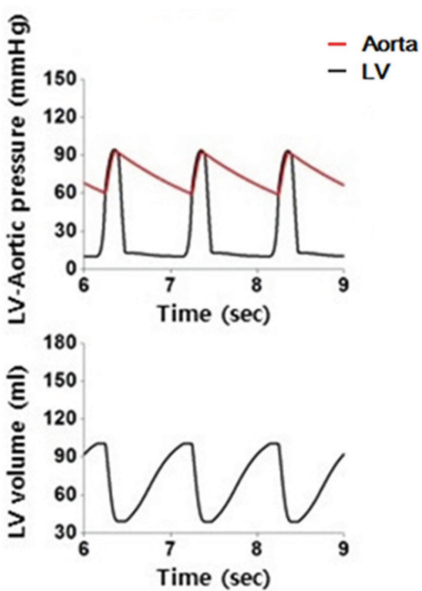

Post-MVCA (6M FU)

Fig. 3. Computed transient solutions of blood pressure and LV volume from the patient-specific pre-MVCA model, post-MVCA $1 \mathrm{M}$ FU model and postMVCA 6M FU model in Case $\mathrm{I}$.

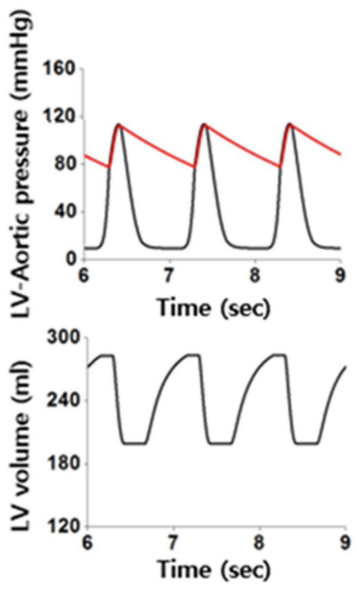

Pre-MVCA
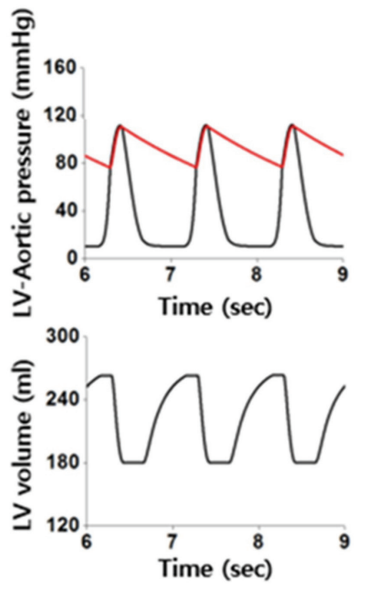

Post-MVCA (1M FU)

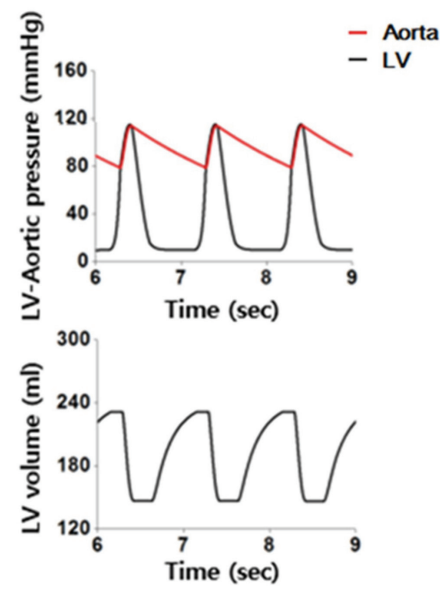

Virtual post-MVCA
Fig. 4. Computed transient solutions of blood pressure and LV volume from the patient-specific pre-MVCA model, post-MVCA $1 \mathrm{M}$ FU model and virtual post-MVCA model in Case II. 


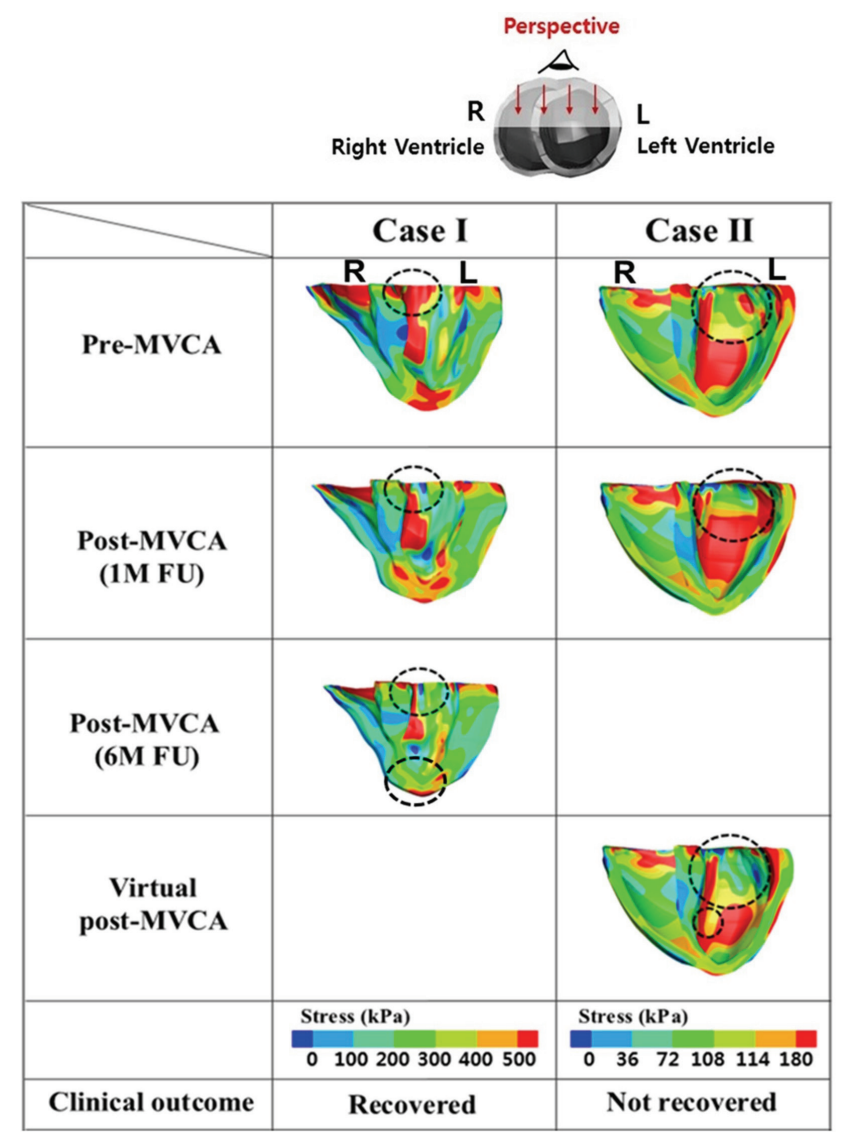

Fig. 5. Ventricular stress distribution of the pre- and post-MVCA models (1-month follow-up (1M FU) and 6-month follow-up (6M FU)) and virtual post-MVCA model in (A) Case I and (B) Case II. Here, stress means von-Mises stress.

ventricular muscle tissue increased from $38 \mathrm{kPa}$ to $40 \mathrm{kPa}$ after MVCA. To propose a physiological hypothesis for the bad clinical outcome of Case II, we tested the virtual post-MVCA model by tightening the diameter of the ventricular basement (Fig. 1). Compared with the pre-MVCA model, a $2 \%$ decrease in diameter was observed in the actual post-MVCA model, while a 7\% decrease in diameter was observed in the virtual post-MVCA model. In the virtual post-MVCA model, the high-stress region clearly decreased at the base and the middle of the ventricle (Fig. 5). Moreover, the mean stress on the ventricular wall decreased significantly after virtual cerclage, from $38 \mathrm{kPa}$ to $35 \mathrm{kPa}$, indicating that tightening of the LV basement can induce stress unloading of the ventricular muscles, as seen in Case II.

\section{DISCUSSION}

MVCA is a less invasive and cost-effective technique for heartfailure patients. However, the clinical outcome is not easily predicted and the MVCA treatment-derived benefits that contribute to cardiac recovery remain unclear. In this sense, cardiac simulation has significant potential for understanding the fundamental physiological mechanism and for outcome prediction of virtual MVCA treatment.

In this paper, we presented virtual cardiac simulations by coupling electrophysiology with an excitation contraction model in pre-MVCA and post-MVCA models. Our aim was to compare the computed values with the clinical values in the pre-MVCA models for clinical validation. We also aimed to understand the biomechanical and physiological mechanism of the stress unloading effect in post-MVCA. Furthermore, a long-term purpose was to apply this technology to optimize a patient-specific cardiac MVCA treatment strategy.

There are three main findings from this study. First, we developed an electromechanical method for a patient-specific ventricle model. To validate the present method, we compared the computed SBP, DBP, EF, EDV, ESV, and MDV values in the patientspecific MVCA ventricle model with clinically measured values. Relatively good agreement between the clinically measured data and the computed results was observed (Tables 3 and 4). The mean differences in SBP, DBP, and EF between the clinical and simulated data were all $\sim 12 \%$ in the pre-MVCA models, while those in the post-MVCA models were $\sim 18 \%$.

Second, a correlation between post-MVCA clinical outcome and MVCA-derived electromechanical changes (Fig. 5) was investigated to explain the underlying mechanism of the benefits of MVCA treatment. The recovery of damaged heart muscle is strongly affected by stress unloading. It is well known that after heart surgery, over time, a lower level of myocardial stress can aid in heart recovery. Compared with the pre-MVCA model, the high-stress region was reduced in the heart-recovered Case I after MVCA. In the recovery-failed Case II, the region was enlarged after MVCA (Fig. 5). After MVCA, the spatially and temporally averaged stress of ventricular muscle tissue was reduced in the clinically heart-recovered Case I, but increased in the clinically recovery-failed Case II. These findings suggest that the reduced region of high stress after MVCA contributes to the electromechanical benefit of the patient-specific ventricular morphology, aiding in heart recovery. This point is also supported by previous studies in which the ventricular shape, size, and wall stress were shown to affect cardiac diseases [14-17].

Lastly, previous animal studies have shown that the tightening degree of the basement cerclage suture is a significant factor for successful heart recovery and must be carefully determined by a patient-specific scheme. As pointed out in the previous paper [2], it is challenging to determine the optimal tightening degree of the mitral septal-lateral dimension. We suggest that the present simulation method be used for this purpose. For example, one of the recovery-failed cases (Case II) was selected and modeled by the present simulation method. Next, we computed the stresses exerted on the ventricular myocardial tissue. According to the simulated results, the stresses were not adequately reduced (i.e., one critical benefit of this cardiac electromechanics simulation is the computation of virtual solutions to predict outcome from 
a virtual MVCA prior to real medical treatment). Thus, this preoperative rehearsal planning tool could be useful in determining the proper tension of the suture line to optimize the MVCA strategy.

However, the present study has several limitations. This simulation is not immediately applicable in clinical practice because the present method was validated only in two typical cases. Many cases should be investigated to validate the present cardiac electromechanics simulations in the actual MVCA model and in the virtual MVCA model. We did not mimic a patient-specific electrophysiological characteristics in these simulations. And the same lumped parameters obtained from pre-MVCA were used for the post-MVCA models. This may be inaccurate in advanced heart disease because the lumped parameters can be affected by the patient-specific cardiac recovery capability after MVCA. In the future, the MVCA-derived cardiac and circulatory conditions will be considered. Despite these limitations, we believe that this simulation has potential for clinical applications by associating influences between electromechanical changes induced by ventricle morphology and subsequent heart recovery. The results from this study suggest that a patient-specific ventricular modeling approach to identify a non-invasive MVCA target radius and stress load on the ventricular muscle tissue prior to the clinical procedure is feasible.

\section{CONCLUSIONS}

We developed a patient-specific electromechanical model of the MVCA treatment by coupling cardiac electrophysiology with heart mechanics. Computed results of this model was compared with clinical data. We found that heart recovery was affected by ventricular stress distribution. Reduced ventricular stresses in the basement after MVCA treatment were observed in the recovered patient model. On the other hand, increased ventricular stresses in the basement after MVCA treatment were found in the recovery-failed patient model. In the recovery-failed case after MVCA, more virtual tightening of the ventricular basement diameter than the actual model can induce stress unloading, aiding in heart recovery. This pre-operative rehearsal planning tool may help to determine the proper tension of the suture line to optimize the MVCA strategy for better clinical performance.

\section{ACKNOWLEDGEMENTS}

This research was supported by the National Research Foundation of Korea grant (NRF-2018R1D1A1B07044528), by a 2-year Research Grant of Pusan National University, and by a Research Grant of Kangwon National University (Project ID: 520150028).

\section{CONFLICTS OF INTEREST}

The authors declare no conflicts of interest.

\section{REFERENCES}

1. Herrmann HC, Maisano F. Transcatheter therapy of mitral regurgitation. Circulation. 2014;130:1712-1722.

2. Kim JH, Kocaturk O, Ozturk C, Faranesh AZ, Sonmez M, Sampath S, Saikus CE, Kim AH, Raman VK, Derbyshire JA, Schenke WH, Wright VJ, Berry C, McVeigh ER, Lederman RJ. Mitral cerclage annuloplasty, a novel transcatheter treatment for secondary mitral valve regurgitation: initial results in swine. J Am Coll Cardiol. 2009; 54:638-651.

3. Kim JH, Sung SC, Chon MK, Kim JO, Lee SH, Lee SY, Je HG, Choo KS, Hwang JM, Kim JS, Park YH, Chun KJ, Kim CM. Mitral loop cerclage as a variant form of mitral cerclage annuloplasty that adds a device (CSTV) for preventing potential complications: a preclinical proof of concept and feasibility study. EuroIntervention. 2016;11:e1669-1679.

4. Park YH, Chon MK, Lederman RJ, Sung SC, Je HG, Choo KS, Lee SH, Shin ES, Kim JS, Hwang KW, Lee SY, Chun KJ, Kim CM, Kim $\mathrm{JH}$. Mitral loop cerclage annuloplasty for secondary mitral regurgitation: first human results. JACC Cardiovasc Interv. 2017;10:597610.

5. Lim KM, Constantino J, Gurev V, Zhu R, Shim EB, Trayanova NA. Comparison of the effects of continuous and pulsatile left ventricular-assist devices on ventricular unloading using a cardiac electromechanics model. J Physiol Sci. 2012;62:11-19.

6. Lim KM, Hong SB, Lee BK, Shim EB, Trayanova N. Computational analysis of the effect of valvular regurgitation on ventricular mechanics using a 3D electromechanics model. J Physiol Sci. 2015;65:159-164.

7. Yuniarti AR, Lim KM. The effect of electrical conductivity of myocardium on cardiac pumping efficacy: a computational study. Biomed Eng Online. 2017;16:11.

8. Trayanova NA, Constantino J, Gurev V. Electromechanical models of the ventricles. Am J Physiol Heart Circ Physiol. 2011;301:H279286.

9. Watanabe H, Hisada T, Sugiura S, Okada J, Fukunari H. Computer simulation of blood flow, left ventricular wall motion and their interrelationship by fluid-structure interaction finite element method. JSME. 2002;45:1003-1012.

10. Kerckhoffs RCP, Healy SN, Usyk TP, McCulloch AD. Computational methods for cardiac electromechanics. Proc IEEE. 2006;94:769783.

11. Gurev V, Lee T, Constantino J, Arevalo H, Trayanova NA. Models of cardiac electromechanics based on individual hearts imaging data: image-based electromechanical models of the heart. Biomech Model Mechanobiol. 2011;10:295-306.

12. ten Tusscher KH, Panfilov AV. Alternans and spiral breakup in a human ventricular tissue model. Am J Physiol Heart Circ Physiol. 2006;291:H1088-1100.

13. Rice JJ, Wang F, Bers DM, de Tombe PP. Approximate model of cooperative activation and crossbridge cycling in cardiac muscle using 
ordinary differential equations. Biophys J. 2008;95:2368-2390.

14. Kono T, Sabbah HN, Stein PD, Brymer JF, Khaja F. Left ventricular shape as a determinant of functional mitral regurgitation in patients with severe heart failure secondary to either coronary artery disease or idiopathic dilated cardiomyopathy. Am J Cardiol. 1991;68:355359.

15. Lorell BH, Carabello BA. Left ventricular hypertrophy: pathogenesis, detection, and prognosis. Circulation. 2000;102:470-479.
16. Sabbah HN, Kono T, Rosman H, Jafri S, Stein PD, Goldstein S. Left ventricular shape: a factor in the etiology of functional mitral regurgitation in heart failure. Am Heart J. 1992;123:961-966.

17. Tibayan FA, Rodriguez F, Langer F, Liang D, Daughters GT, Ingels NB Jr, Miller DC. Undersized mitral annuloplasty alters left ventricular shape during acute ischemic mitral regurgitation. Circulation. 2004;110(11 Suppl 1):II98-102. 